Proceedings of the International School and Conference on Optics and Optical Materials, ISCOM07, Belgrade, Serbia, September 3-7, 2007

\title{
"Necklace-Ring" Beams in Saturable Kerr Media with Square-Root Nonlinearity
}

\begin{abstract}
M.S. Petrović*
Institute of Physics, Pregrevica 118, 11080 Belgrade, Serbia

Analytical and numerical investigation of the propagation of "necklace-ring" optical beams in Kerr-like saturable photorefractive media with square-root nonlinearity is carried out. Analytic expression for the propagation dynamics of the necklace beams is in good agreement with numerical findings.
\end{abstract}

PACS numbers: 42.65.Tg, 42.65.Sf

\section{Introduction}

Progress in generating spatial optical solitons in nonlinear (NL) bulk media opens the possibility of intense study of two-dimensional (2D) interaction and selftrapping of light beams [1]. Recently, very interesting soliton structure in the form of azimuthally periodically modulated beams ("necklace beams") was reported in $[2,3]$. Self-trapped necklace beams can exist in a homogeneous bulk NL optical medium, exhibiting quasi-stable expansion even in a self-focusing NL medium [2-4]. Because of zero angular momentum they do not rotate during propagation. In this paper, we investigate propagation of necklace-ring optical beams in Kerrlike saturable photorefractive (PR) media with square-root nonlinearity.

We utilize an isotropic model of PR media with a local interaction of beams that is especially suitable for transverse $2 \mathrm{D}$ geometries. The model developed in $[5,6]$ contains a more realistic expression for the PR space-charge field of the form $E_{\mathrm{SC}}=E_{0} / \sqrt{1+I}$, where $E_{0}$ is the transverse dc electric field applied to the PR crystal, and $I$ is the total beam intensity measured in units of the dark or background intensity. The square-root intensity dependence represents more accurately the most relevant isotropic contribution to the space-charge field and constitutes an improved model to be used for describing the propagation of $(2+1) \mathrm{D}$ spatial screening solitons. It offers more accurate results in comparison to the straightforward generalization of the 1D formula $1 /(1+I)$, which is used by most of authors. In addition, it leads to improved stability of propagating complex optical structures and longer propagation distances [7].

*e-mail: petrovic@phy.bg.ac.yu 


\section{Theory}

The equation for the slowly varying optical field envelope $\psi$ can be written in the form of the general dimensionless NL Schrödinger equation

$$
\mathrm{i} \frac{\partial \psi}{\partial z}+\Delta_{\perp} \psi-\frac{1}{\sqrt{1+|\psi|^{2}}} \psi=0
$$

where $\Delta_{\perp}$ is the transverse Laplacian and $z$ is the propagation distance, measured in the units of the diffraction length $L_{\mathrm{D}}$. Following the standard procedure, the action of the root model is easily found

$$
S=\int_{-\infty}^{\infty}\left[\frac{\mathrm{i}}{2}\left(\psi \frac{\partial \psi^{*}}{\partial z}-\text { c.c. }\right)+\left|\nabla_{\perp} \psi\right|^{2}+2 \sqrt{1+|\psi|^{2}}\right] \mathrm{d} x \mathrm{~d} y \mathrm{~d} z .
$$

We utilize this quantity in describing the propagation of necklace-ring optical beams in the medium. The procedure is to determine optimal parameters for different beam structures utilizing variational principles, then launch optimized beams into the medium, and observe the subsequent behavior.

Following the idea and notation given in [3], we consider a necklace beam described by an input ansatz

$$
\psi(r, \theta, z)=\frac{\alpha(z) \cos (\Omega \theta) \mathrm{e}^{-\mathrm{i} \Gamma(z) z+\mathrm{i} v(z) r}}{\cosh (a(z)(r-L(z)))},
$$

where $\alpha$ is the amplitude, $\Omega$ is a half of the number of the pearls, $1 / a$ is the ring thickness, and $L$ is the necklace radius. Necklace radius is large compared to radial thickness $(L \gg 1 / a)$; another parameter restriction is necklace stability requirement $\Omega \gg L \pi a / 4[3]$. The main quantity characterizing a spatial soliton is its power $E=\iint|\psi|^{2} \mathrm{~d} x \mathrm{~d} y=2 \pi \alpha^{2} L / a$, which is dimensionless here, and represents an integral of motion. Because of the energy conservation relation, the only parameters to be optimized are $\alpha(z), L(z)$, and $v(z)$. Optimal values of these parameters can be found from the principle of minimum action. Propagation of necklace beam with initial shape given by Eq. (3), whose parameters are determined by the least action principle, is shown in Fig. 1.
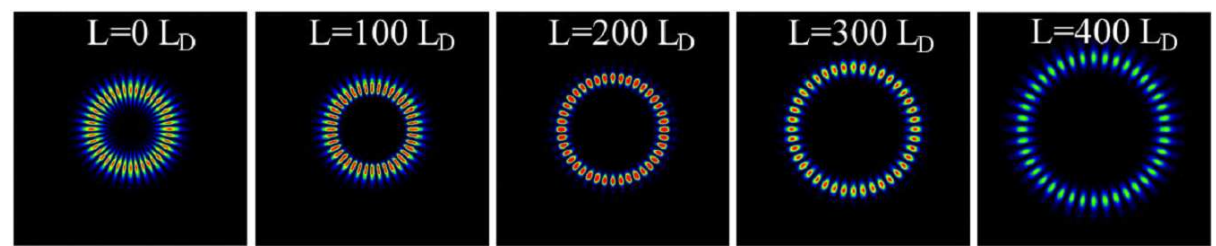

Fig. 1. Example of typical evolution of a necklace beam: the necklace slowly expands during propagation. For all plots, the (transverse) axes are the same.

We substitute the ansatz (3) into the relation for the action (Eq. (2)), and integrate the resulting expression in transverse directions in the manner described in [3], which yields 


$$
\begin{aligned}
S= & \int_{-\infty}^{\infty} \mathrm{d} z\left[\frac{\partial v}{\partial z} L E-E \frac{\partial(\Gamma z)}{\partial z}+v^{2} E+\frac{4 \pi^{2} \alpha^{4} L^{2}}{3 E}+\frac{\Omega^{2} E}{L^{2}}\right. \\
& \left.+\int_{0}^{2 \pi} \mathrm{d} \theta \int_{0}^{\infty} r \mathrm{~d} r 2 \sqrt{1+\psi \psi^{*}}\right] .
\end{aligned}
$$

One can notice that only the last term must be solved approximately.

\section{Small amplitude approximation}

First, we consider a small amplitude approximation. In this case $\sqrt{1+\psi \psi^{*}} \approx$ $1+\frac{|\psi|^{2}}{2}-\frac{|\psi|^{4}}{8}$, and action is given by

$$
S=\int_{-\infty}^{\infty} \mathrm{d} z\left[\frac{\partial v}{\partial z} L E-E \frac{\partial(\Gamma z)}{\partial z}+v^{2} E+\frac{4 \pi^{2} \alpha^{4} L^{2}}{3 E}+\frac{\Omega^{2} E}{L^{2}}+E-\frac{\alpha^{2} E}{8}\right]
$$

The minimization of the action $\partial S / \partial \alpha=\partial S / \partial L=\partial S / \partial v=0$ gives next relations:

$$
\alpha^{2}=\frac{3}{64} \frac{E^{2}}{\pi^{2} L^{2}}, \quad 2 \frac{\partial v}{\partial z}=\frac{4 \Omega^{2}-3 E^{2} / 256 \pi^{2}}{L^{3}}, \quad \text { and } \quad \frac{\partial L}{\partial z}=2 v .
$$

From the first relation it follows $a=\frac{\sqrt{3}}{4} \alpha$; using additional two relations we find the following expression for the necklace radius $L$ :

$$
L(z)=\sqrt{\left[\frac{1}{L_{0}^{2}}\left(4 \Omega^{2}-\frac{3}{256} \frac{E^{2}}{\pi^{2}}\right)+4 v_{0}^{2}\right] z^{2}+4 L_{0} v_{0} z+L_{0}^{2}},
$$

where the subscript zero denotes the initial conditions at $z=0$.

We execute a series of numerical simulations of different necklaces, using a beam propagation method based on the fast Fourier transform technique. We find excellent agreement between observed numerical necklace-ring dynamics and our analytical analysis founded on the least action principle (Fig. 2).

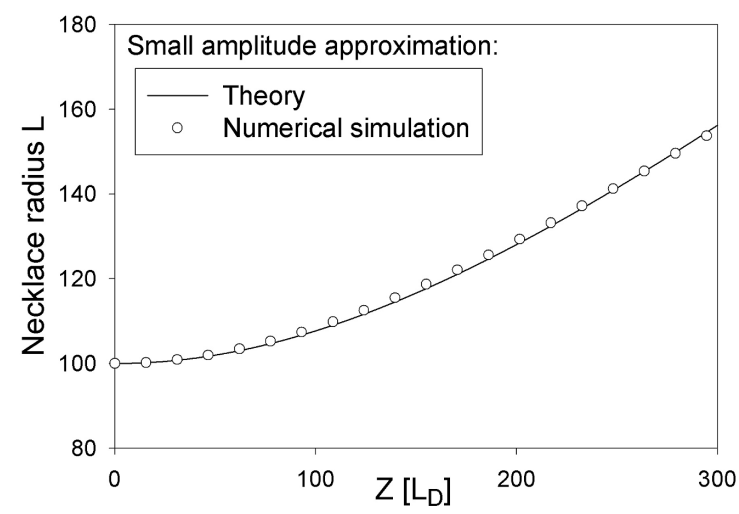

Fig. 2. Necklace radius as a function of propagation distance in the small amplitude approximation: comparison between numerical beam-propagation results and analytical predictions. Input necklace parameters: $\alpha=0.1, a=(\sqrt{3} / 4) \alpha=0.0433, L=100$, $\Omega=20$. 


\section{Large amplitude approximation}

Next, we consider a large amplitude approximation. In this case $\sqrt{1+\psi \psi^{*}} \approx|\psi|$, and action is given by

$$
S=\int_{-\infty}^{\infty} \mathrm{d} z\left[\frac{\partial v}{\partial z} L E-E \frac{\partial(\Gamma z)}{\partial z}+v^{2} E+\frac{4 \pi^{2} \alpha^{4} L^{2}}{3 E}+\frac{\Omega^{2} E}{L^{2}}+\frac{4 E}{\alpha}\right] .
$$

The minimization of the action $\partial S / \partial \alpha=\partial S / \partial L=\partial S / \partial v=0$ gives next relations:

$$
\alpha^{5}=\frac{3}{4} \frac{E^{2}}{\pi^{2} L^{2}}, \quad 2 \frac{\partial v}{\partial z}=\frac{4 \Omega^{2}}{L^{3}}-4\left(\frac{4 \pi^{2}}{3 E^{2}}\right)^{1 / 5} \frac{1}{L^{3 / 5}} \approx \frac{4 \Omega^{2}}{L^{3}}, \quad \text { and } \quad \frac{\partial L}{\partial z}=2 v .
$$

The second term in the second relation can be neglected because it is much smaller than the first, as a consequence of the necklace stability requirement $L \gg 1 / a \gg L \pi / 4 \Omega$. From the first relation it follows $a=\sqrt{3 / \alpha}$; using additional two relations we find the following expression for the necklace radius $L$ :

$$
L(z)=\sqrt{\left(\frac{4 \Omega^{2}}{L_{0}^{2}}+4 v_{0}^{2}\right) z^{2}+4 L_{0} v_{0} z+L_{0}^{2}} .
$$

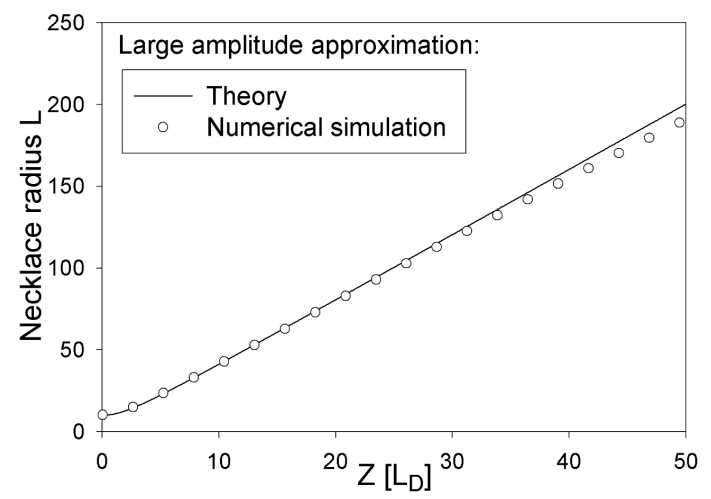

Fig. 3. Necklace radius as a function of propagation distance in the large amplitude approximation: comparison between numerical beam-propagation results and analytical predictions. Input necklace parameters: $\alpha=10, a=\sqrt{3 / \alpha}=0.548, L=10, \Omega=20$.

We execute a series of numerical simulations, as before. We find excellent agreement between the observed numerical necklace-ring dynamics and our analytical analysis (Fig. 3).

\section{Conclusion}

We investigated analytically and numerically propagation of necklace-ring optical beams in Kerr-like saturable photorefractive media with square-root nonlinearity. We obtained analytic expressions for the propagation dynamics of the necklace beams in small/large amplitude approximations, in good agreement with 
numerical simulations. Results in this paper open possibilities for investigation of interaction-collision phenomena between self-trapped necklaces and other solitons.

\section{Acknowledgments}

This work was supported by the Ministry of Science of the Republic of Serbia, under grant number 141031.

\section{References}

[1] Yu.S. Kivshar, G.P. Agrawal, Optical Solitons, Academic Press, San Diego 2003.

[2] M. Soljačić, S. Sears, M. Segev, Phys. Rev. Lett. 81, 4851 (1998).

[3] M. Soljačić, M. Segev, Phys. Rev. E 62, 2810 (2000).

[4] A.S. Desyatnikov, Yu.S. Kivshar, Phys. Rev. Lett. 87, 033901 (2001).

[5] G.F. Calvo, F. Agulló-López, M. Carrascosa, M. Belić, W. Królikowski, Europhys. Lett. 60, 847 (2002).

[6] G.F. Calvo, F. Agulló-López, M. Carrascosa, M.R. Belić, D. Vujić, Opt. Commun. 227, 193 (2003).

[7] M. Petroski, M. Petrović, M. Belić, Opt. Commun. 279, 196 (2007). 